\title{
Traveling towards fame: Albert Einstein and the Eddington eclipse expedition to Príncipe and Sobral in 1919
}

Lapo Casetti

\section{Introduction}

Albert Einstein is the icon of modern science. His name is the first that comes to everyone's mind to be put next to the word «scientist». With his discoveries Albert Einstein revolutionized our concepts of space and time. Time magazine named Albert Einstein «person of the century» in its December 31, 1999 issue. What is less known, however, is that Albert Einstein abruptly became famous all over the world, and not only among fellow scientists, essentially in just a few days near the end of 1919. And what especially matters for the subject of this volume is that the origin of his fame is rooted in a journey of some eminent British scientists to remote locations where the Portuguese language was (and still is) spoken, to observe a total eclipse of the Sun; a journey that started in the spring of 1919 . Thus, in 2019 we all celebrated five hundred years since Magellan departed for his journey around the globe as well as fifty years since a journey into outer space allowed a man to set foot on another celestial body for the first time, but the physics community also celebrates a hundred years since this less known journey took place. The following pages are devoted to the story of that journey, of the experiments that were performed and of their consequences, and to a discussion of what came next, until a few months ago. But before departing to our remote destination, let us recall the historical and scientific background.

Lapo Casetti, University of Florence, Italy, lapo.casetti@unifi.it, 0000-0002-6964-5611

FUP Best Practice in Scholarly Publishing (DOI 10.36253/fup_best_practice)

Lapo Casetti, Traveling towards fame: Albert Einstein and the Eddington eclipse expedition to Príncipe and Sobral in 1919, pp. 421-440, (c) 2021 Author(s), CC BY 4.0 International, DOI 10.36253/978-88-5518467-0.34, in Michela Graziani, Lapo Casetti, Salomé Vuelta García (edited by), Nel segno di Magellano tra terra e cielo. II viaggio nelle arti umanistiche e scientifiche di lingua portoghese e di altre culture europee in un'ottica interculturale, (C) 2021 Author(s), content CC BY 4.0 International, metadata CC0 1.0 Universal, published by Firenze University Press (www.fupress.com), ISSN 2704-5919 (online), ISBN 978-88-5518467-0 (PDF), DOI 10.36253/978-88-5518-467-0 


\section{Lights all askew in the heavens: Einstein's abrupt rise to fame}

In 1919 Albert Einstein is a highly respected professor in Berlin, is one of the most important physicists of the world and an influential personality in the German-speaking cultural environment. His most important contributions to science have already been published. Yet, he is largely unknown to the general public. But things are going to change. On November 7, 1919, The Times of London publishes an article entitled Revolution in science. New theory of the Universe. Newton's ideas overthrown (the ideas of the greatest British scientist of all times overthrown by a German scientist, barely one year after the end of World War I!). Three days after, a column of The New York Times opens with the memorable title Lights all askew in the Heavens; and continues, wittily enough, Einstein theory triumphs. Stars not where they seemed or were calculated to be, but nobody need worry. And again, British scientist called the discovery one of the greatest of human achievements. The two Times are the most important newspapers of the world: word spreads rapidly and Albert Einstein abruptly becomes the most famous scientist of the globe. It is one of the first documented cases where mass media make a piece of news travel around the globe in a few days and change the world's perception on a subject (Coles 2001; Kennefick 2019; Will 2015). Who is the British scientist mentioned by The New York Times? What happened at the beginning of November 1919, to arouse the interest of the two influential newspapers? What is this scientific discovery that would be one of the greatest of human achievements? And above all, what are these lights all askew in the Heavens?

The British scientist is Arthur Stanley Eddington (who will become Sir Arthur, but has not been knighted yet), one of the leading astrophysicists of the beginning of the twentieth century. The two newspapers reported on the meeting of the Royal Society of November 6, 1919 in London, where Eddington presented the results of the measurements performed by the team led by the Astronomer Royal Frank Dyson and by himself during the total solar eclipse of May 29, 1919, observed from the island of Príncipe in equatorial Africa and from Sobral in northern Brazil. These measurements confirmed a prediction of Einstein's general theory of relativity, namely, that light rays are bent when passing close to a large mass: during the eclipse, stars close to the Sun's limb appeared displaced with respect to their position in the sky when the Sun was not in that area of the sky (these are the lights that are all askew in the Heavens), and the (tiny) amount of displacement was that predicted by Einstein. It was the smoking gun proving Einstein's theory right. To better appreciate the story, let us step back and briefly discuss the science behind Einstein's prediction.

\section{The general theory of relativity and gravitational light bending}

Albert Einstein presented the final version of his general theory of relativity to the Prussian Academy of Sciences in late 1915. The paper containing the 
full exposition of the theory and the correct ${ }^{1}$ prediction of the light bending by the Sun appeared a few months later in the Annalen der Physik, the leading German-language physics journal of the times (Einstein 1916). The theory had been the result of ten years of intense work, mainly carried out by Einstein alone, but for an important collaboration with his friend, the mathematician Marcel Grossman (Einstein and Grossmann 1913, 1914). The general theory of relativity is undoubtedly one of the greatest achievements of science: it has completely changed our conception of space and time, replacing the absolute space and absolute time of Newtonian physics with a dynamic spacetime, where space and time are not only unavoidably mixed but are no longer the static stage where physical events happen, becoming themselves changing and evolving objects. Among the main consequences of general relativity is the realization that gravity is not a force exerted by one body on another one, as earlier described by Newton, but a property of spacetime itself. In general relativity, gravity is nothing but the curvature of spacetime; such a curvature is due to mass and energy. Although the mathematical language of differential geometry is needed to fully describe the theory, in order to understand the origin of the light bending effect one may think that in a flat spacetime both matter particles and light rays would travel in straight lines, but when a large concentration of mass or energy is present, it distorts the fabric of spacetime in such a way that matter particles and light rays have to move on curved trajectories. The larger the mass or energy, the larger the effect is on trajectories of particles and light rays. According to this picture, the Earth orbits the Sun because the straight line it would follow in the absence of the Sun is distorted into an elliptical orbit by the curvature of spacetime. Now, consider a light ray coming from a very distant star and grazing the Sun before being observed on the Earth:

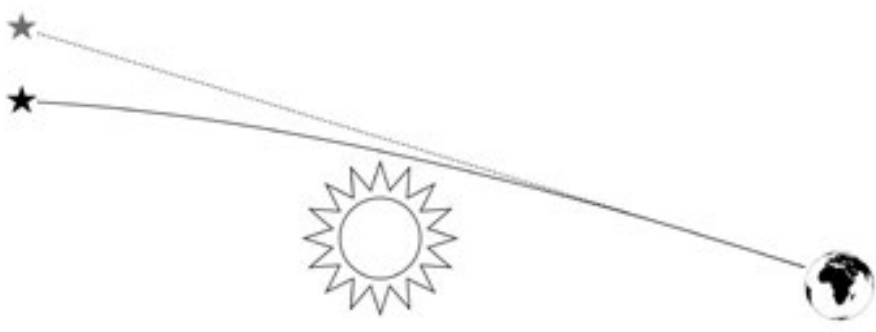

Fig. 1 - Sketch of the gravitational light bending effect (Credit: L. Casetti).

1 As we shall see in the following, Einstein had put forward a prediction of the light bending by the Sun already some years before; but such a prediction was wrong, amounting to one half of the correct result. 
passing close to the Sun its trajectory is bent, so that, when we observe the light, the apparent position of the star it comes from is shifted from its real position, i.e., from its position on the sky it normally has when the Sun is not close to it. In other words, the Sun acts as a 'gravitational lens'. The amount of displacement, as calculated by Einstein in 1915, is 1.75 seconds of arc. One second of arc is $1 / 3600$ of a degree: as a comparison, the full Moon spans nearly half a degree in the sky, that is 1800 seconds of arc. Then, the displacement of the apparent position of the star is definitely a tiny quantity: it roughly corresponds to the apparent size of a 50 Euro cent coin seen from a distance of two kilometers. This notwithstanding, it was a measurable quantity even in the early twentieth century: astronomers had learned through the centuries to measure the positions of celestial objects with remarkable accuracy, made even better by the recent (at the times) addition of photography to the astronomer's toolbox. A photograph of the night sky taken through a telescope, if made with suitable precautions and accompanied by other calibration photographs, would suffice to measure star positions up to the precision needed to reveal the effect predicted by Einstein, but there is the big problem that when the Sun is up in the sky stars cannot be seen.

In a letter from Zürich dated October 14, 1913, Einstein writes to the famous American astronomer George Ellery Hale to ask him whether, using a telescope, a star could be observed sufficiently near the Sun to reveal the effect, during the day or maybe during a total eclipse of the Sun, when the Moon covers for a few minutes the disk of the Sun and stars appear in the sky. Hale answers that the daylight measurement is impossible even with the most powerful telescopes ${ }^{2}$ but that the measurement during an eclipse is surely possible.

The reader may be confused with the dates, and a clarification is in order. We said that Einstein performed his calculation of the light bending effect in 1915 , as an application of the general theory, but the letter to Hale dates back to 1913. Which effect is Einstein referring to, then? Indeed, Einstein predicted that gravity should bend the paths of light rays already in 1911 (Einstein 1911), as a consequence of his «equivalence principle», stated in 1907 (Einstein 1907), that is the starting point of the general theory of relativity, but is not sufficient to determine the correct equations describing the gravitational field. The equivalence principle essentially states that there is no local experiment that allows to distinguish between a gravitational field and uniformly accelerated motion with respect to an inertial frame of reference. Einstein told that such a principle was suggested to him by the realization that if one is freely falling in a gravitational field, then does not feel his own weight, and later $^{3}$ referred to this realization as

2 Indeed, the brightest stars can be easily seen and photographed during the day with a telescope, but this is much more difficult when they are close to the Sun, because the diffuse light from the Sun is overwhelming the fainter light of the star.

3 As stated in note 2 of (Einstein 2002), this writing, famous for the «happiest thought», was probably a draft of an invited article for Nature written in 1920 and aiming at describing the general theory of relativity to non-specialized readers, that was however never published because Nature editors deemed it too long. 
«the happiest thought of my life» (Einstein 2002). It must be stressed that such a statement may appear more or less obvious to us, that are used to watch videos of astronauts weightlessly floating in the International Space Station, that indeed are in free fall in the Earth's gravity field. But clearly no such videos were available to Einstein! And no experimental realization of a free fall was easily conceivable at the beginning of the twentieth century, because it requires, if not a true spacecraft, at least an airplane flying on a parabolic trajectory, like those used for the training of astronauts and for shooting space scenes in Hollywood movies. This is one of the neatest examples of the ability of Einstein to invent Gedankenexperimenten (thought experiments) to illustrate aspects of the physical reality difficult to reproduce in a laboratory. According to the equivalence principle, light must 'fall' in a gravity field as if it were made of massive particles. However, the 1911 prediction by Einstein of the amount of light bending due to the Sun was wrong because it did not take into account the fact that the geometry of space is not flat (Comer and Lathrop 1978; Ehlers and Rindler 1997), resulting in the prediction of a smaller effect (actually, one half of the correct value: 0.875 arc seconds). Being entirely based on the equivalence principle, the 1911 prediction was equivalent to assuming that light moves in a gravitational field as if it were made of material particles following the Newton's laws of motion: therefore, it is commonly referred to as the 'Newtonian prediction'. It was later realized that such a prediction had already been published by the German astronomer Johann Georg von Soldner as early as in 1801 (von Soldner 1801) and that essentially the same calculation had been performed (but not published) by Henry Cavendish some twenty years before von Soldner (Will 1988), Einstein being unaware of both these previous calculations.

\section{Measuring light bending by the Sun during total eclipses}

Already in 1911 Einstein had started contacting astronomers to convince them to try to measure the light bending effect. Both American and European scientists were interested, and the one who really took up the task with enthusiasm was a young scientist based in Berlin, Erwin Freundlich. He also suggested to Einstein to investigate the possibility of performing the measurements by photographing bright stars close to the Sun in broad daylight (Kennefick 2019). But after Hale's answer to Einstein, it appeared that total eclipses might be the only key to an experimental verification of light bending by the Sun. Various astronomers had looked at available photographic plates taken during eclipses to see whether they could be used to this purpose, but the smallness of the effect was such that no available images were useful: data had to be carefully collected with this precise measurement in mind, if one wanted to have a reasonable chance to measure light bending during an eclipse. The total eclipse occurring on August 21, 1914, seemed a remarkable opportunity: it could be observed from Europe, since the path of totality ran from North-west to South-east touching (referring to nowadays countries) Norway, Sweden, Finland, Latvia, Lithuania, Belarus, Ukraine, the Black Sea, Turkey, Iraq, and Iran. An expedition of German astro- 
nomers, including Freundlich, departed to Crimea to observe the eclipse and try to measure light bending a couple of months before the date of the eclipse itself. But soon World War I began, and the German scientists were arrested and interned as citizens of an enemy country (and possible spies). Freundlich managed to come back to Germany after an exchange of prisoners in September 1914, but the opportunity was lost ${ }^{4}$. One may say, in hindsight, that Einstein was 'lucky' that the measurement could not take place, given that in 1914 he had only derived the wrong prediction yet; but using the word «luck» in connection with a devastating war seems out of place.

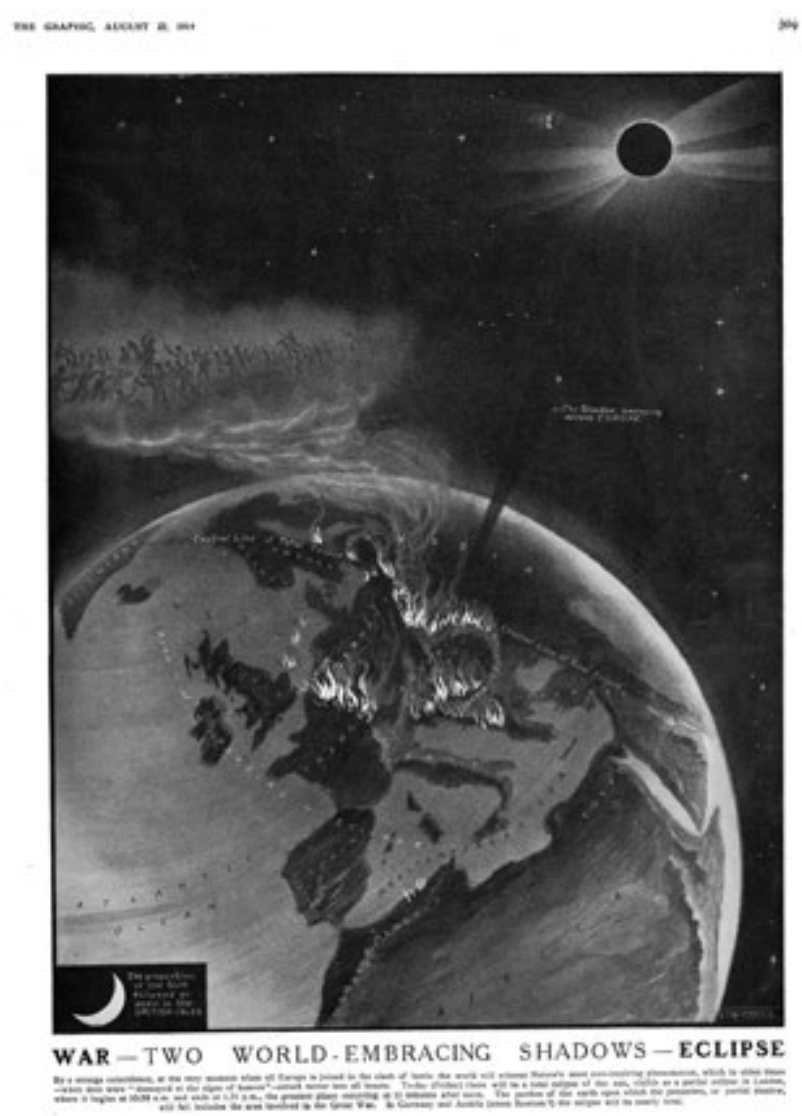

Fig. 2 - An illustration appeared on The Graphic of London on August 22, 1914, showing Europe immersed in the two shadows of eclipse and war (Credit: from the collection of Michael Zeiler, www.eclipse-maps.com. See also Dickinson 2014).

4 For more details see (Kennefick 2019), Chapter 4. 


\subsection{The 1919 eclipse expedition to Príncipe and Sobral}

With World War I drawing to an end, the two main characters (besides Einstein) of our story enter the scene: Frank Watson Dyson, Astronomer Royal (director of the Greenwich Observatory) and the already mentioned Arthur Stanley Eddington, then professor at Cambridge and director of the Observatory of the same University. Dyson and Eddington are the two most important British astronomers of the times: Dyson is an 'old style' astronomer, with a great observational experience, while Eddington is the pioneer of the new astrophysics: he will contribute to lay the foundations of this discipline. He is an expert in the general theory of relativity and a great admirer of Einstein. Dyson and Eddington realize that the total eclipse of May 29, 1919, is a unique opportunity (Eddington 1919). Totality will be very long (more than five minutes) and a lot of bright stars belonging to the Hyades cluster will appear around the eclipsed Sun in the Taurus constellation. No situation as favorable as this will occur in decades. At variance with the 1914 eclipse, however, a journey to remote locations is unavoidable: the only landfalls of the totality path are in South America and in Africa.

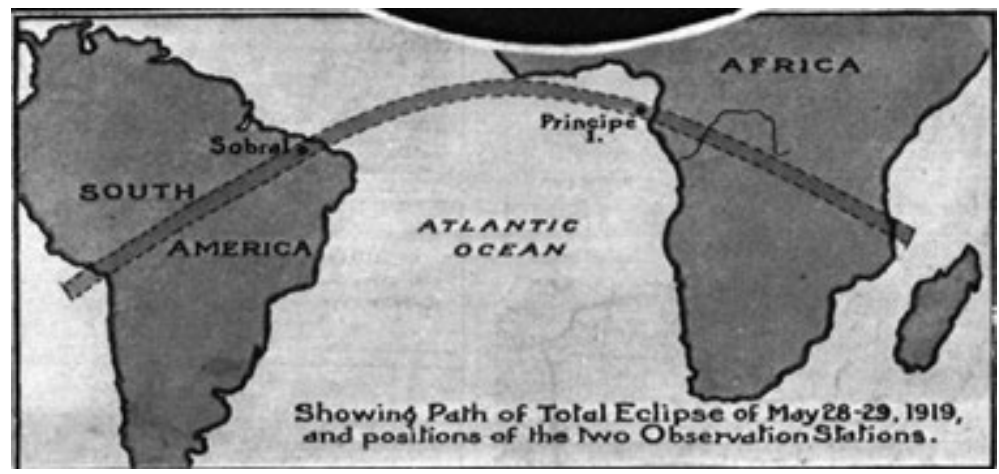

Fig. 3 - Map of the path of the May 29, 1919 solar eclipse, highlighting the two destinations of the 1919 expedition (Credit: adapted by the author from an illustration appeared on the Illustrated London News, November 22, 1919, and found in the collection of Michael Zeiler, www.eclipse-maps.com).

Dyson and Eddington decide to organize two coordinated expeditions: the Greenwich Observatory expedition, led by Dyson (who would however remain in England, and will later coordinate the analysis of the Sobral data) and composed of Andrew Crommelin and Charles Davidson will travel to Sobral, in northern Brazil; the Cambridge Observatory expedition, including Eddington himself and the technician Edwin Cottingham, will reach the island of Príncipe, in the Gulf of Guinea, some $250 \mathrm{~km}$ off the coast of Gabon in equatorial Africa. Both destinations speak Portuguese: Brazil had been a colony of the Kingdom of Portugal from 1500 and until 1822; Príncipe was still a colony of Portugal in 1919. The Republic of São Tomé and Príncipe, having gained independency 
from Portugal in 1975, is nowadays the smallest Portuguese-speaking country in the world. After loading telescopes and other tools, the two expeditions depart on March 8, 1919 from Liverpool onboard the RMS Anselm. The first stop is in Madeira, where the two expeditions separate: Davidson and Crommelin continue towards Belém in Brazil, while Eddington and Cottingham stay in Madeira for nearly a month, waiting for a ship to Príncipe. In a letter ${ }^{5}$ to his family, Eddington states that it was «a wonderful holiday». On April 9 the Portugal ship departs for Príncipe, where Eddington and Cottingham arrive two weeks later.

Meanwhile Crommelin and Davidson have reached Sobral, where they have received a warm welcome by the Brazilian scientists. Weather is well-promising, climate is dry. The observing station is set up: two refracting telescopes are laid horizontal and fixed, and light is fed to their objective lenses by two coelostats, i.e., two flat moving mirrors able to follow the apparent motion of objects in the sky thanks to a clockwork mechanism.

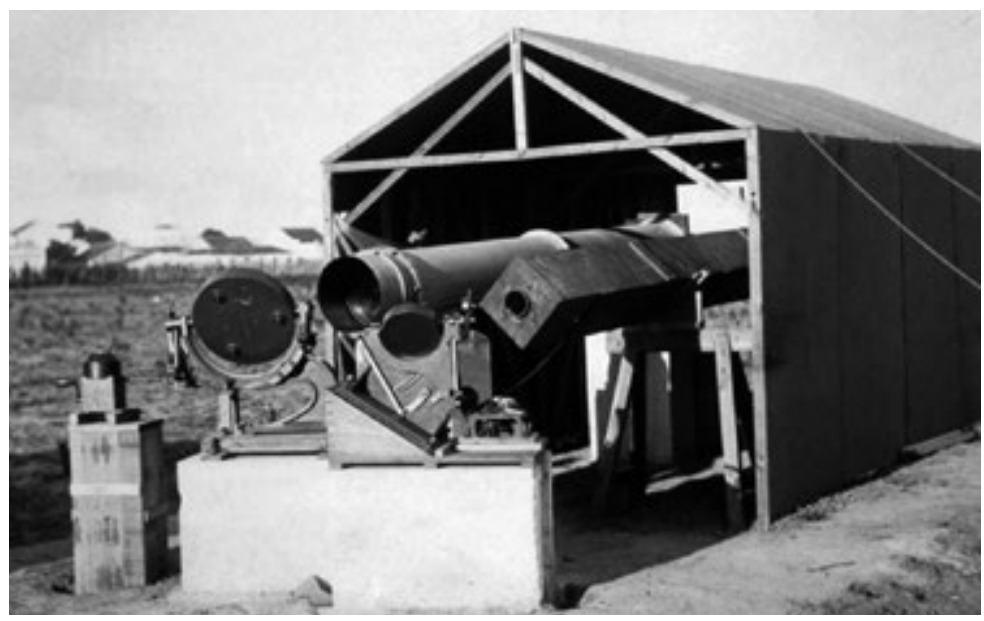

Fig. 4 - The observing station of Davidson and Crommelin in Sobral. The large telescope on the left is the $25 \mathrm{~cm}$, while the smaller one is the $10 \mathrm{~cm}$. The two coelostats are visible in front of the telescopes, outside the hut (Credit: photo by Charles Davidson, courtesy of Graham Dolan, The Royal Observatory, Greenwich, UK).

The largest telescope has a $25 \mathrm{~cm}$ objective lens, while the smaller one is equipped with a $10 \mathrm{~cm}$ lens. The $25 \mathrm{~cm}$ telescope is the main instrument and is expected to yield the best results but will suffer serious problems, due to deformations of the large coelostat mirror: data obtained with this telescope will be discarded in the end. The smaller telescope, originally meant as a backup, will be the one delivering the best results of both expeditions, as we shall discuss below. 
Arrived in Príncipe, Eddington learns from the locals that good weather at the end of May is very unlikely, and that clouds tend to gather close to the mountains, so that he looks for a place far from the mountainous terrain and chooses a cocoa plantation in the northern part of the island, Roça Sundy. Today there is a commemorative plaque and a hotel that can be reserved online, but no pictures of the Eddington observing station have remained. On eclipse day, May 29, it rains nearly until the beginnings of the eclipse, then intermittent clouds are present during all the phenomenon, disturbing the observation. Eddington goes nonetheless through the planned observation, exposing many photographic plates, but only two of them will turn out to be of sufficient quality to be used for the measurement of star positions. Sobral plates, thanks to far better atmospheric conditions, are of a much greater quality.

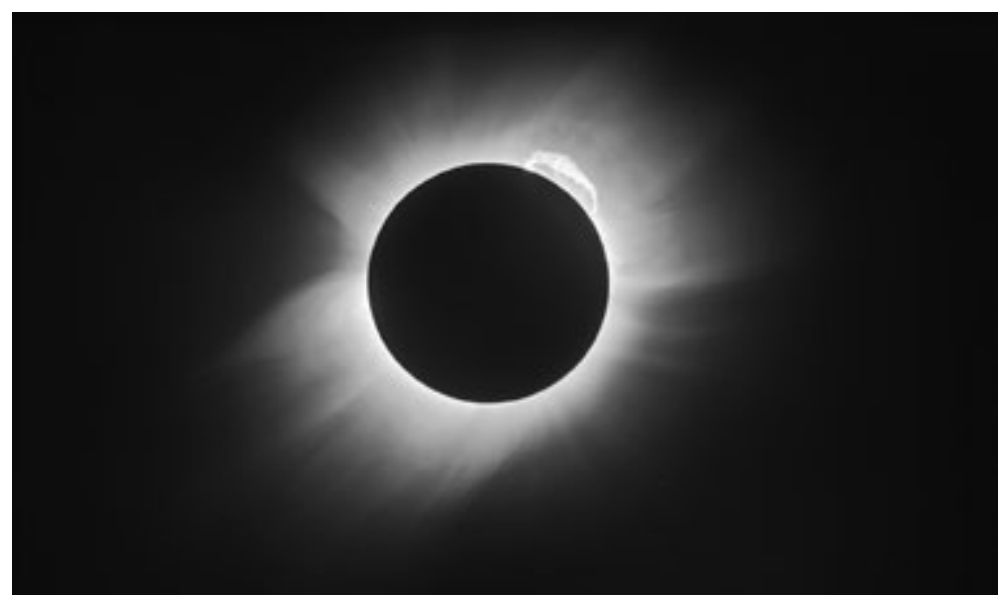

Fig. 5 - Image of the Sun during the total solar eclipse of May 29, 1919, from a photographic plate taken with the $10 \mathrm{~cm}$ telescope in Sobral and processed with modern techniques (Credit: ESO/Landessternwarte Heidelberg-Königstuhl/F. W. Dyson, A. S. Eddington, and C. Davidson).

For a first-hand account of the preparation of the expedition, of the journey, of the eclipse day measurements and of the data reduction see the scientific paper describing the results (Dyson et al. 1920).

\subsection{Data analysis and results}

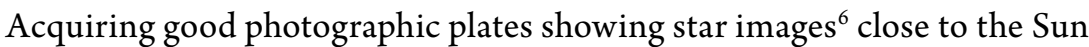
is just the starting point. In order to measure the shift in star positions due to

6 More precisely, the light distribution resulting from the combined effect of the diffraction due to the optical system and of the blurring due the atmosphere on the star image formed on the focal plane of the telescope. 
gravitational deflection, photographs of the same star field taken with the same instrument when the Sun is not there but the conditions are as much as possible the same as during the eclipse are needed ${ }^{7}$. To acquire such comparison star field images, Davidson and Crommelin stay in Sobral after the eclipse until the end of July, to wait until the Taurus constellation appears at the end of the night nearly as high in the sky as during the eclipse of May 29. Eddington had instead acquired the comparison photographs before, from Oxford, with the same telescope he would then use in Príncipe.

With both teams back in England, the data analysis begins and is, as expected, long and difficult. The gravitational light deflection effect, if present, is small: the arrow in figure 6 shows the shift of the center of a star image on a Sobral plate. Looking at this picture the measurement of such a small shift seems impossible, but it must be stressed that the "blob" representing the star image on the plate is not of uniform intensity, and with a careful measurement of the variation of the intensity it is possible to pinpoint the position of the center of the star up to a fraction of an arcsecond. The most important parameter to estimate, together with the star positions, is just the uncertainty of the measure, the crucial parameter to assess whether experimental data are or not consistent with the theoretical predictions. In 1919 Einstein had corrected his prediction of the light bending using the full theory of general relativity so that three different outcomes are considered as possible according to different theoretical approaches: no gravitational deflection at all, the 'Newtonian' deflection of 0.875 arcseconds, and the Einstein prediction of 1.75 arcseconds of deflection (all these values refer to a star located exactly at the solar limb: for more distant stars the effect falls off proportionally to the distance from the center of the Sun). A relative accuracy of no more than some tens of percent is needed to discriminate between the alternatives. This would be 'easy' for a measurement performed at an observatory, but this is not an observatory measure at all. As already stated by Eddington before departing to Príncipe,

This in itself calls for no extravagant precautions of accuracy; but the main difficulties arise from the awkward conditions of eclipse observations (Eddington 1919).

Data from the two expeditions are analyzed separately: Dyson is in charge of the analysis of the Sobral plates, while Eddington supervises the reduction of the data acquired by himself in Príncipe. Only at the end of the analysis the results from the two data reduction processes will be put together to obtain the final result. This is best practice in physics experiments: for instance, a similar procedure has been used in the discovery of the Higgs boson at CERN in 2012,

7 This was especially true in 1919 when the positions of stars in the sky were not known $a$ priori with a sufficient accuracy. Today such comparison photographs would not be strictly necessary, although they still may be used in the data analysis of a similar experiment, if available (see the discussion of the TAROT experiment below). 


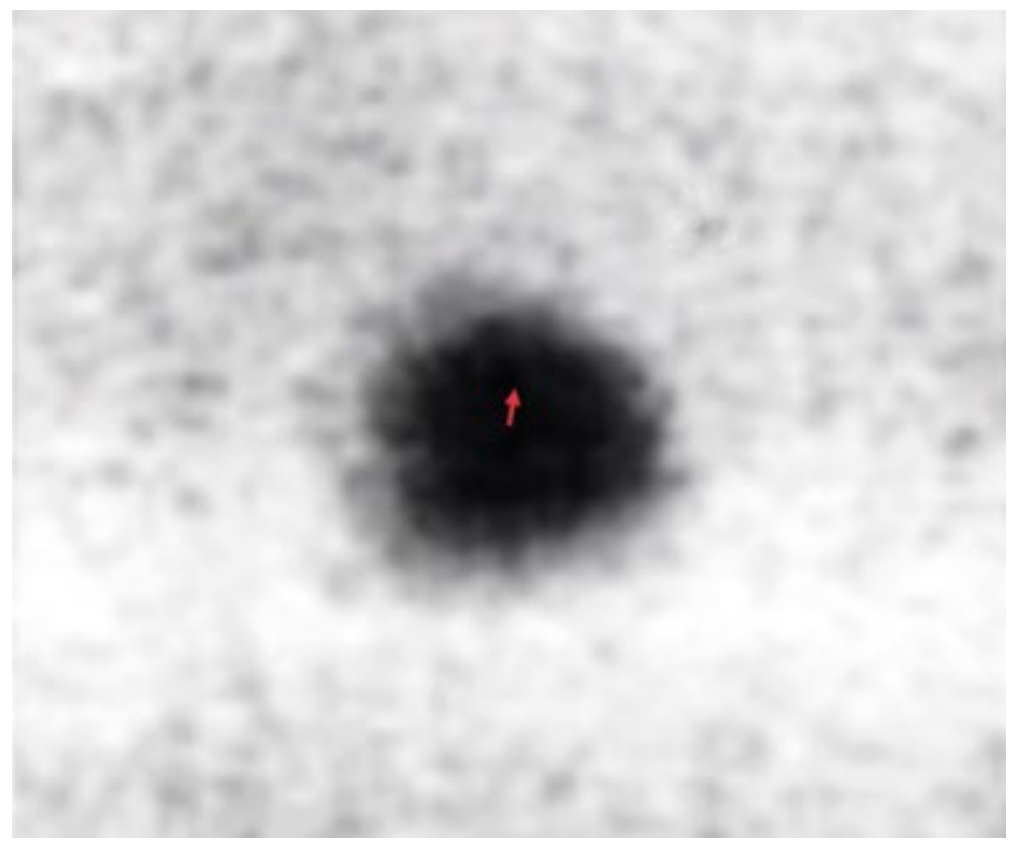

Fig. 6 - Enlargement of the negative image of one of the stars on a photographic plate taken with the $10 \mathrm{~cm}$ telescope in Sobral. The star is roughly at 2.3 solar radii from the center of the Sun, and the arrow indicates the amount of displacement of the center of the star according to Einstein's prediction for the gravitational light bending, that is, 0.75 arc seconds (Credit: courtesy of Robin Catchpole, The Royal Observatory, Greenwich, UK).

where the data from two separate experiments (ATLAS and CMS) have been separately analyzed and put together only at the end (ATLAS collaboration 2012; CMS collaboration 2012). During the analysis of the Sobral data, it turns out that the plates taken with the $25 \mathrm{~cm}$ telescope are not reliable. The problem is probably due to the fact that the large coelostat mirror suffered deformations as a consequence of the temperature variation between the partial and the total phases of the eclipse. Images were deemed nearly unusable by the observers after development of the plates ${ }^{8}$. Luckily enough, the $10 \mathrm{~cm}$ telescope, meant as a backup, performed nearly flawlessly. A thorough description of the data

8 The scientific paper describing the expedition and the results (Dyson et al. 1920) quotes the following note by Davidson and Crommelin, taken on the night of May 30 soon after developing the plates: «It was found that there had been a serious change of focus, so that, while the stars were shown, the definition was spoilt. This change of focus can only be attributed to the unequal expansion of the mirror through the sun's heat. [...] It seems doubtful whether much can be got from these plates». 
analysis is given in (Dyson et al. 1920), where it is reported that the measured deflection obtained by the $10 \mathrm{~cm}$ telescope plates in Sobral is 1.98 arc seconds, with an estimated uncertainty of \pm 0.12 arc seconds, while the Príncipe observations yield a deflection of 1.61 arc seconds, with an estimated uncertainty of \pm 0.30 arc seconds ${ }^{9}$; the two independent results are thus perfectly consistent. The Sobral measurements made with the $25 \mathrm{~cm}$ telescope yield a deflection of 0.93 arc seconds, but with a much larger uncertainty that is difficult to estimate, being mostly due to systematic effects as the defocusing mentioned above. In the end, Dyson decides that the results from the $25 \mathrm{~cm}$ telescope have to be kept separate and not combined with the others, considering only the results coming from plates made with the $10 \mathrm{~cm}$ backup telescope as trustworthy results of the Sobral expedition ${ }^{10}$. All the results, including the 'bad' ones, clearly rule out the absence of deflection, while the two most reliable ones also rule out the 'Newtonian' deflection, definitely pointing at the correctness of Einstein's prediction. The paper concludes:

\begin{abstract}
Thus the results of the expeditions to Sobral and Principe can leave little doubt that a deflection of light takes place in the neighbourhood of the sun and that it is of the amount demanded by Einstein's generalised theory of relativity, as attributable to the sun's gravitational field. But the observation is of such interest that it will probably be considered desirable to repeat it at future eclipses. The unusually favourable conditions of the 1919 eclipse will not recur, and it will be necessary to photograph fainter stars, and these will probably be at a greater distance from the sun (Dyson et al. 1920).
\end{abstract}

\title{
4.3. Einstein, general relativity, and light bending in the media
}

The results of the 1919 eclipse expedition to Sobral and Príncipe were presented by Eddington at a meeting of the Royal Society in London on November 6, 1919. The rest is known: the titles of The Times and The New York Times we mentioned at the beginning appeared, and Einstein became famous all over the world. In the following years Einstein and his theory of relativity were often featured in the news, but also in movies. A particularly relevant, although not widely known, example is a documentary movie written by Garrett P. Serviss and produced in 1923 by the studios ran by Max and Dave Fleischer (who was also the director), the authors of famous cartoon characters as Popeye and especially Betty Boop. Such a movie, entitled Einstein theory of relativity, was essentially a remake of the previous (and unfortunately lost) German movie Die Grundlage der Einsteinschen Relativitäts-Theorie directed in 1922 by the Ger-

9 The weighted average of the two results yields a deflection at the solar limb of $1.91 \pm 0.11 \mathrm{arc}$ seconds, that agrees with Einstein's prediction to within $1.5 \sigma$. The relative uncertainty of the measurement is of $6 \%$.

10 See (Kennefick 2019), Chapter 12. 
man scientist-director Hanns Walter Kornblum, who will also direct in 1925 the masterpiece Wunder der Schöpfung (Wonders of creation), a summary of the astronomical knowledge of the 1920's. The Serviss-Fleischer 1923 movie contains an excellent illustration for a general audience of the phenomenon of light bending and of its observation during an eclipse that is still effective today, despite its vintage appearance.

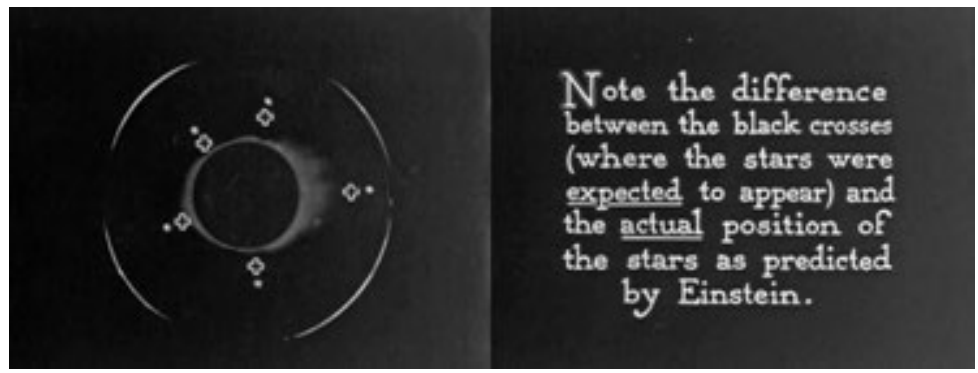

Fig. 7 - Stills from the documentary movie The Einstein theory of relativity (G. P. Serviss, D. Fleischer, M. Fleischer 1923) illustrating the shift in the positions of stars close to the Sun during an eclipse due to gravitational light bending (Credit: adapted by the author from the footage of the movie, that can be found for instance at https:// vimeo.com/9832926).

\subsection{After the 1919 expedition: from 1922 until today}

As predicted by Eddington, there have been various attempts at repeating the measurement of light bending made during the 1919 eclipse. The first one was an American expedition to observe the September 21, 1922 eclipse in Australia (Campbell 1923), led by the Lick Observatory director W.W. Campbell. The final results were in excellent agreement with Einstein's prediction, the quoted result for the deflection at the solar limb being $1.75 \pm 0.09$ arc seconds (Campbell and Trumpler 1928). However, results were not published until six years after the eclipse, because the data analysis turned out to be very difficult and the result, despite the much better equipment (that did no longer include the coelostats that had created so many problems before) and the fact that nearly a hundred stars' positions were measured, was essentially as precise as the Dyson-Eddington 1919 one, the relative uncertainty being of $5 \%$ while that of the 1919 measurement was of $6 \%$. But better precision proved very hard to be obtained even in the attempts to come in the following years. Erwin Freundlich had his own opportunity during the 1929 eclipse: he employed a novel calibration technique that allegedly should have solved the difficulties related to the change of scale between eclipse and comparison fields, one of the main source of uncertainty in the 1919 and 1922 measurements. Freundlich claimed to have measured a deflection larger that 2 arc seconds: this figure, however, was later corrected after a careful re-analysis of his data made by R.J. Trumpler and converged to the Einstein value of 1.75 arc 
seconds, with an accuracy of the same order of the previous ones. Other attempts followed ${ }^{11}$, among which those by the Soviet astronomer Aleksandr Mikhailov (who observed the 1936 and 1941 eclipses in the former Soviet Union and later the 1952 eclipse in Brazil) and by the Yerkes Observatory astronomer George van Biesbroeck (who observed the 1947 and 1952 eclipses; in the latter he met Mikhailov); both independently tried another way to overcome the calibration difficulties but did not succeed in improving the precision of the results. The last big expedition to attempt an 'Eddington experiment', as the measurement of light deflection at a solar eclipse had been nicknamed, was the American expedition organized by Princeton University and the University of Texas to observe the June 30, 1973 eclipse at the Chinguetti Oasis in Mauritania. Despite the very big effort, several things went wrong and the final result was in agreement with Einstein, but with a relative uncertainty even worse than the previous ones, around 10\% (Brune et al. 1976; Jones 1976). After more than fifty years, the 5\% bound on attainable precision still resisted. After 1973, for nearly another fifty years nobody tried to repeat an Eddington experiment, at least using visible light. Indeed, any electromagnetic radiation, and not only visible light, is affected by the gravitational bending effect. It was then realized that measuring the deflection of radio waves by the Sun, instead of radiation at visible wavelength, was much more promising in terms of accuracy, because radio waves emitted by celestial bodies can be detected during the day as well as during the night, so that there is no longer any need to wait for a rare event like a total solar eclipse, that in addition requires traveling to remote locations where no established observatories are typically present ${ }^{12}$ : moreover, one could use the then-recently-discovered quasars as sources of radio waves, since they are pointlike like a star (although we now know that their radiation comes from the active nuclei of faraway galaxies). With this technique gravitational deflection of radio waves was soon measured with accuracies around 1\% (Fomalont and Sramek 1977), and modern refinements have improved this figure by more than three orders of magnitude; comparable accuracies in the optical band have then been reached with spacebased observations, especially with the Hipparcos astrometric satellite, and the new European GAIA astrometric satellite promises to improve the accuracies by two further orders of magnitude (Will 2015). Needless to say, all results are in perfect agreement with Einstein's theory: this is one of the rare cases in science where a theoretical prediction has resisted the improving of the precision of measurements by many orders of magnitude.

Today it is apparent (as it already was at the end of the 1970s) that solar eclipses are no longer needed to test Einstein's general relativity. This notwithstanding, the question of whether one could perform an Eddington experiment during a total eclipse and significantly improve the precision overcoming the ' $5 \%$ wall' remained open and resurfaced in recent years, given that new technologies li-

11 See e.g. (Kennefick 2019), Chapter 14.

12 But see note 13 below. 
ke the introduction of CCD detectors had completely changed the scenario of astronomical observations with respect to 1973, when photographic plates were still in use. The challenge was taken by Donald G. Bruns, a retired American physicist who carefully planned and rehearsed for two years an Eddington experiment to be carried out during the total solar eclipse of August 21,2017, known as the 'great American eclipse' since the path of totality swept the continental US coast-to-coast from Oregon to South Carolina. Bruns performed the experiment in Wyoming on his own, using only off-the-shelf high-end portable amateur equipment: a big change with respect to the 1973 expedition which set up a temporary astronomical observatory in the Sahara and was composed of many scientists with different specializations. Bruns' experiment was highly successful, demonstrating that with careful planning a single person can now obtain better results than a big expedition, making use of the available technology. The deflection at the solar limb measured by Bruns was $1.7512 \pm 0.0595$ arc seconds (Bruns 2018), in perfect agreement with Einstein's prediction and with a relative precision of $3.4 \%$, the best to date for an Eddington experiment, nearly a factor of two better than the pioneering 1919 measurement and well below the 5\% threshold. It must be noted that Bruns could exploit a far superior technology with respect to Eddington and his coworkers in 1919, but the conditions of the 2017 eclipse were definitely worse in terms of duration of the totality (only two minutes) and number of bright stars close to the Sun: had he had comparable conditions he would probably have attained an even better accuracy. Thanks to the availability of very precise star positions in nowadays catalogues, Bruns could avoid using comparison star fields and introduced some smart techniques for data acquisition and calibration that might be very useful to repeat the experiment in the future with similar equipment: although, as already discussed, no new scientific knowledge is expected to be gained from performing an Eddington experiment at future solar eclipses, such experiments might have a high educational value and could be attempted, for instance, by groups of physics and astronomy students.

Exactly one hundred years after the historical 1919 eclipse, another solar eclipse occurred on July 2, 2019, and was a really unique opportunity, because the European Southern Observatory (ESO) facilities at La Silla, in northern Chile, were in the path of totality.

It is very rare that a total solar eclipse can be observed from a professional observatory $\mathrm{y}^{13}$, let alone one hosting some of the most advanced instruments of the world and with exceptionally good observing conditions as La Silla. The

13 However, it occurred more often than one would expect. For instance, totality swept over professional telescopes in 1991 at the Mauna Kea observatory in Hawai'i and in 1961 at the Observatoire de Haute-Provence in France and at two observatories in Italy (the Loiano observatory halfway between Bologna and Florence and the Arcetri Observatory in Florence, the latter being very close to the centerline of the totality path). Although the telescopes on Mauna Kea were used for scientific observations in 1991, no Eddington experiments were performed at the observatories in the paths of totality of the 1991 and 1961 eclipses, to the best of our knowledge. 


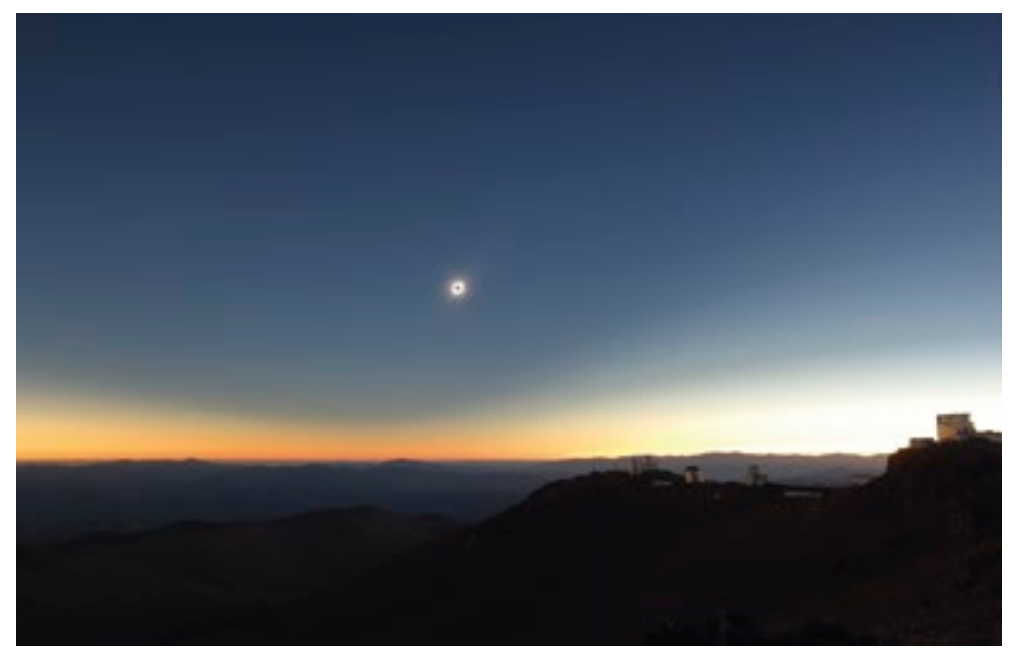

Fig. 8 - Wide-angle view from the ESO-La Silla observatory towards the eclipsed Sun and the Pacific Ocean during the total phase of the July 2, 2019 solar eclipse. Several telescope domes are visible on the right: the largest one is that of the New Technology Telescope that was pointed at the solar corona to perform spectroscopic observations (Credit: L. Casetti).

opportunity was not missed: ESO organized a highly successful public viewing event at La Silla, and various scientific observations were performed using telescopes of the Observatory. Among the latter, the TAROT telescope was selected to attempt an Eddington experiment: for the first time, star positions close to the Sun were measured during a total eclipse with a professional telescope in a permanent location. A movie on the 2019 TAROT experiment is available online (Doyen 2019). At variance with Bruns, the TAROT team decided to go for a data reduction workflow which uses comparison star fields acquired six months after the eclipse, in January 2020, when the stars were exactly at the same height in the sky as during the eclipse; the COVID-19 pandemic has then slowed down all the process, so that the data analysis is not complete yet (Klotz 2020). We still have to wait to see the final results and to know whether the data taken one hundred years after the historic 1919 eclipse will turn out to be the most precise in a century.

\section{Concluding remarks}

If the Sun can act as a gravitational lens, deflecting light rays coming from distant stars, larger masses can act as even more powerful gravitational lenses. Indeed, a galaxy or a cluster of galaxies can deflect the light coming from more distant galaxies, creating multiple images of the same galaxy and even distorting their images 
into single of multiple arches ${ }^{14}$. Moreover, focusing the images as an optical lens, would do, they allow us to study faint and distant galaxies that would otherwise be impossible to see; conversely, from the shape of the distorted image, it is possible to estimate the mass distribution of the object acting as a lens (Bartelmann 2010). Gravitational lenses have become a fundamental tool in modern astrophysics and can be considered - together with discovering the smoking gun proving Einstein right - the most important heritage of the 1919 eclipse expedition.

Any story of the 1919 expedition to Príncipe and Sobral would be incomplete without mentioning the century-long debate on the alleged unreliability of the 1919 measurements due to a bias of Eddington in favor of Einstein: in short, during the years rumors spread about the fact that Eddington would have pushed the results of his and the Greenwich's group towards agreement with Einstein's prediction, by underestimating uncertainties of the Príncipe and Sobral $10 \mathrm{~cm}$ telescope observations and forcing his coworkers to discard the results obtained with the Sobral $25 \mathrm{~cm}$ telescope which seemed to point more towards the 'Newtonian' value of the deflection. Eddington would have been dishonest, and the true first reliable confirmation of Einstein's prediction would have been that coming from the $1922 \mathrm{ex}-$ pedition to Australia led by Campbell, whose results were published only in 1928 (Campbell and Trumpler 1928). The reason for Eddington's bias would have been twofold: his admiration of Einstein and of his theory, and the fact that he shared Einstein's pacifist ideas, so that he thought a confirmation by British scientists of a theory put forward by a German scientist soon after the war would help the peace cause. Such rumors survived until today and even eminent scientists like the late Stephen Hawking supported them (Hawking 1988). In the opinion of the author, Kennefick's discussion of the subject (Kennefick 2009,2012,2019) completely clarifies the issue, very convincingly arguing that the alleged Eddington's bias is nothing but a myth. In the following we just highlight some points that, in the author's opinion, convincingly suggest that there was no bias in Eddington's conclusions drawn from the 1919 data, referring the interested reader to Kennefick's papers (Kennefick 2009, 2012) and book (Kennefick 2019) for a deeper discussion. One of the arguments issued by those who believe the 1919 results were not reliable is that Campbell and Trumpler's 1928 results obtained by reducing the 1922 data are intrinsically more trustworthy because Campbell did not believe Einstein's prediction and cannot be suspected of a bias towards Einstein, at variance with Eddington. But many scientific experiments have been planned and performed just to confirm theoretical predictions: think of the already mentioned search for the Higgs boson at the LHC or of the detection of gravitational waves with the LIGO and Virgo interferometers (LIGO and Virgo collaborations 2016), just to mention two recent Nobel-prize-worthy results. If all the results obtained by scientists who devoted years of work - if not an entire career - to designing, planning, and performing an experiment should be considered unreliable just because the scientists belie-

14 A perfect alignement between a pointlike source, a spherical mass and the observer would yield a circular image of the source. 
ved their experiment would be successful, we could even stop doing science right now. Then, as we already mentioned, the decision of discarding the data obtained with the $25 \mathrm{~cm}$ telescope in Sobral (one of the 'proofs' of Eddington's dishonesty for some supporters of the 'conspiracy') was not made by Eddington, who played no role in analyzing the Sobral data that belonged to the Greenwich team, but by Dyson, who was not a strong Einstein supporter. Simply put, Dyson had very good reasons as an expert observer to reject data coming from an instrument that had a poor performance and were found in disagreement with those obtained at the same time and in the same place with an instrument which performed nearly flawlessly. A 'mild' version of the myth tells that Eddington was not dishonest, but simply lucky: according to this story the real precision of the 1919 measurements was far worse than quoted, but the result just luckily fell close to Einstein's prediction and a not-so-careful analysis of the uncertainties allowed Eddington to state that the measurements confirmed Einstein's theory, while he should have declared that no conclusive result had been obtained because the uncertainties were too big. However, also this argument was shown to be wrong. In 1979 the photographic plates taken in Sobral in 1919 were re-analyzed at the Royal Greenwich Observatory using more precise instruments than those available in 1919, finding that not only the uncertainty on the $10 \mathrm{~cm}$ telescope plates measurements was slightly smaller than that estimated in 1919, but also that the then-discarded measurements obtained with the $25 \mathrm{~cm}$ telescope were perfectly consistent with the others and with Einstein's prediction, although with a larger uncertainty, and much less consistent with the 'Newtonian' deflection, although the latter would not be totally ruled out on a statistical basis by the $25 \mathrm{~cm}$ plates alone. The 1979 results for the light deflection at the solar limb were $1.90 \pm 0.11$ arc seconds for the $10 \mathrm{~cm}$ telescope (to be compared with the result of $1.98 \pm 0.18$ arc seconds found after the 1919 analysis) and $1.55 \pm 0.34$ arc seconds for the $25 \mathrm{~cm}$ telescope (Harvey 1979; Kennefick 2009).

One may wonder why this myth proved so resistant along a century and is still alive. A possibility, especially concerning the 'mild' version, is again suggested by Kennefick in his book (Kennefick 2019). One would naively expect that by repeating an experiment many times its accuracy should considerably increase and not stay essentially the same or even get worse as it happened in the case of the Mauritanian eclipse expedition, whence the belief that if in 1973 only a $10 \%$ accuracy was obtained, then the real accuracy of the 1919 results should have been much worse than the claimed $6 \%$. However, there are two reasons why the accuracy of an experiment usually improves over the years: first, a significant technology advance may intervene, and second, hands-on knowledge accumulates with trial and error. The Mauritanian eclipse team was using essentially the same kind of technology used by the 1919 expedition both for data acquisition and for data reduction (the latter being witnessed by the fact that the 1979 re-analysis of the 1919 data yielded essentially the same results as the 1919 data reduction), so that the sought-after accuracy improvement could come only from the planned measurement of many more star positions than any previous expedition. Unfortunately, at the end 150 positions were measured, a number similar to that of the 1922 expedition and much smaller than the 1500 planned; moreover, many of 
them were not sufficiently close to the Sun to significantly contribute to the final result. On the other hand, it is very difficult to accumulate hands-on knowledge at eclipse measurements of gravitational light bending, because the location, the environmental conditions, and the equipment itself are always different, so that each attempt is more or less as performing an experiment for the first time.

A final lesson to be learned from this story concerns the importance of redundancy and backups in designing and performing an experiment, because something can go wrong even in the most carefully planned experiments. Had the Greenwich astronomers not performed measurements also with the smaller telescope in Sobral, the whole expedition would have been a near-failure, because only on the basis of the measurements made in Sobral with the $25 \mathrm{~cm}$ telescope and in Príncipe by Eddington no really convincingly conclusive discrimination between the Einstein's and the 'Newtonian' predictions for the gravitational light deflection would have been possible. The instrument originally meant as a backup was the key to success.

\section{Acknowledgments}

I wish to explicitly mention Daniel Kennefick's excellent book No shadow of a doubt (Kennefick 2019), a masterful account of the story of the 1919 eclipse expedition; I strongly recommend Kennefick's book to anyone who wants to know more about many of the subjects discussed in this paper. Moreover, I thank Giulio Peruzzi for drawing my attention to Will's paper on the Cavendish calculation (Will 1988). Finally, I warmly thank Alain Klotz for sharing with me information on preliminary results of the 2019 TAROT measurements.

\section{Riferimenti bibliografici}

ATLAS collaboration. 2012. "Observation of a new particle in the search for the Standard Model Higgs boson with the ATLAS detector at the LHC”. Physics Letters B 716: 1 .

Bartelmann, M. 2010. “Gravitational lenses.” Classical and Quantum Gravity 27: 233001. Brune, R.A., et al. (The Mauritanian Eclipse Team). 1976. "Gravitational deflection of light: Solar eclipse of 30 June 1973 I. Description of procedures and final results." The Astronomical Journal 81: 452.

Bruns, D.G. 2018. "Gravitational starlight deflection measurements during the 21 August 2017 total solar eclipse.” Classical and Quantum Gravity 35: 075009.

Campbell, W.W. 1923. "The Total Eclipse of the Sun, September 21, 1922.” Publications of the Astronomical Society of the Pacific 35: 11.

Campbell, W.W., and R. J. Trumpler. 1928. "Observations made with a pair of five-foot cameras on the light-deflections in the Sun's gravitational field at the total eclipse of September 21, 1922.” Lick Observatory bulletin 397: 130.

CMS collaboration. 2012. "Observation of a new boson at a mass of $125 \mathrm{GeV}$ with the CMS experiment at the LHC.” Physics Letters B 716: 30.

Coles, P. 2001. "Einstein, Eddington and the 1919 Eclipse", in "Historical Development of Modern Cosmology." ASP Conference Proceedings 252: 21.

Comer, R.P., and J.P. Lathrop. 1978. "Principle of equivalence and the deflection of light by the sun." American Journal of Physics 46: 801. 
Doyen, G. 2019. “Dans l'ombre de Einstein.” YouTube video <https://youtu.be/ q9D6iyUYNDE> (12/20).

Dickinson, D. 2014. "Remembering the 'World War I Eclipse'” Universe Today August 20, 214 <https://www.universetoday.com/113882/remembering-the-world-war-ieclipse/ $>(12 / 20)$.

Dyson, F.W., A.S. Eddington, and C. Davidson. "A determination of the deflection of light by the Sun's gravitational field, from observations made at the total eclipse of May 29, 1919.” Philosophical Transactions of the Royal Society A 220: 291 (1920).

Eddington, A.S. 1919. "The total eclipse of 1919 May 29 and the influence of gravitation on light." The Observatory 42: 119.

Ehlers, J., and W. Rindler. 1997. "Local and global light bending in Einstein's and other gravitational teorie." General Relativity and Gravitation 29: 519.

Einstein, A. 1907. "Über das Relativitätsprinzip und die aus demselben gezogenen Folgerungen." Jahrbuch der Radioaktivität und Elektronik 4: 411.

Einstein, A. 1911. "Über den Einfluß der Schwerkraft auf die Ausbreitung des Lichtes." Annalen der Physik 35: 898.

Einstein, A. 1916. “Die Grundlage der allgemeinen Relativitätstheorie.” Annalen der Physik 49: 769.

Einstein, A. 2002. "Fundamentals and methods of the theory of relativity, presented in its development." Collected papers of Albert Einstein, vol 7: 113 (English translation supplement). Princeton: Princeton University Press.

Einstein, A., and M. Grossmann. 1913. "Entwurf einer verallgemeinerten Relativitätstheorie und einer Theorie der Gravitation." Zeitschrift für Mathematik und Physik 62: 225.

Einstein, A., and M. Grossmann. 1914. "Kovarianzeigenschaften der Feldgleichungen der auf die verallgemeinerte Relativitätstheorie gegründeten Gravitationstheorie.” Zeitschrift für Mathematik und Physik 63: 215.

Fomalont, E.B., and R.A. Sramek. 1977. "The deflection of radio waves by the Sun." Comments on Astrophysics 7: 19.

Harvey, G.M. 1979. "Gravitational deflection of light: a re-examination of the observations of the solar eclipse of 1919.” The Observatory 99: 195.

Hawking, S. 1988. A brief history of time. London: Bantam Press.

Jones, B.F. 1976. "Gravitational deflection of light: Solar eclipse of 30 June 1973 II. Plate reductions." The Astronomical Journal 81: 455.

Kennefick, D. 2009. “Testing relativity from the 1919 eclipse-a question of bias.” Physics Today 62: 37.

Kennefick, D. 2012. "Not Only Because of Theory: Dyson, Eddington, and the Competing Myths of the 1919 Eclipse Expedition.” In Einstein and the Changing Worldviews of Physics, eds. C. Lehner, J. Renn, and M. Schemmel, Einstein Studies, vol. 12, Boston: Birkhäuser.

Kennefick, D. 2019. No shadow of a doubt. Princeton: Princeton University Press.

Klotz, A. 2020. private communication.

LIGO and Virgo collaborations. 2016. "Observation of Gravitational Waves from a Binary Black Hole Merger.” Physical Review Letters 116: 061102.

von Soldner, J. 1801. Berliner Astronomisches Jahrbuch. 161 (1801-1804), reprinted in von Soldner, J. (Lenard, P.). 1921. “Über die Ablenkung eines Lichtstrahls von seiner geradlinigen Bewegung durch die Attraktion eines Weltkörpers, an welchem er nahe vorbeigeht; von J. Soldner 1801.” Annalen der Physik 65: 593.

Will, C.M. 2015. "The 1919 measurement of the deflection of light." Classical and Quantum Gravity 32: 124001. 